\title{
Pellet Embolization after Subclavian Artery Injury: A Case Report
}

\author{
Raminta Černevičiūtė1, Shaheel M. Sahebally², Ahmad Mourad33, Donatas Inčiūra33, \\ Aleksandras Antuševas ${ }^{3}$
}

${ }^{1}$ Faculty of Medicine, Lithuanian University of Health Sciences, Kaunas, Lithuania

${ }^{2}$ Department of Surgery, University College Hospital Galway, Galway, Ireland

${ }^{3}$ Department of Cardiac, Thoracic and Vascular Surgery, Lithuanian University of Health Sciences,

Hospital of Lithuanian University of Health Sciences Kaunas Clinics, Kaunas, Lithuania

Email: cerneviciute.raminta@gmail.com

How to cite this paper: Černevičiūtè, R., Sahebally, S.M., Mourad, A., Inčiūra, D. and Antuševas, A. (2017) Pellet Embolization after Subclavian Artery Injury: A Case Report. Surgical Science, 8, 444-450.

https://doi.org/10.4236/ss.2017.810049

Received: October 3, 2017

Accepted: October 24, 2017

Published: October 27, 2017

Copyright (c) 2017 by authors and Scientific Research Publishing Inc. This work is licensed under the Creative Commons Attribution International License (CC BY 4.0).

http://creativecommons.org/licenses/by/4.0/

(c) (i) Open Access

\begin{abstract}
Acute upper limb ischemia caused by a gunshot penetrating vascular injury and subsequent arterial embolization by foreign body is uncommon in everyday practice and is associated with high morbidity/mortality rates, especially with emergency surgery. We present the case of a 72-year old male who attempted suicide using a gun. A gunshot entry wound was evident in the right upper chest region with no pellet exit wound. Radial pulses were palpable bilaterally. Angiography revealed right subclavian artery direct injury and pellet embolization to the brachial artery. The patient underwent open surgery, with reversed saphenous vein interposition graft to replace subclavian artery defect. A pellet was removed by a separate arteriotomy in the brachial artery. Associated injuries were clavicle-comminuted fracture and subclavian vein injury. The patient remained well 5 months later with no reported complications. In conclusions, the presence of radial pulses alone on clinical exam cannot rule out the presence of a significant vascular injury.
\end{abstract}

\section{Keywords}

Gunshot Wound, Pellet Embolization, Arterial Trauma, Upper Extremity, Case Report

\section{Introduction}

Penetrating vascular injuries are associated with high mortality rates, with a significant percentage of patients dying before reaching the hospital [1] [2]. Moreover, gunshot vascular injuries may be associated with bone, nerve and muscu- 
lotendinous damage and treating these complex injuries usually requires a multidisciplinary team approach [3]. As patients with intra-arterial pellet migration may be asymptomatic, because of a good collateralization, this may delay diagnosis and subsequently result in poor outcomes. Therefore a rapid diagnosis for a physician can be challenging. According to Feliciano D.V. et al., patients after penetrating trauma, presenting with symmetrical pulses and normal ABPI, should be discharged and followed-up. Only $1 \%-4 \%$ of these asymptomatic patients eventually undergo surgery [4]. We report a rare case of a pellet embolization to the right brachial artery after right upper chest gunshot injury. The patient had concomitant subclavian artery and vein injury and comminuted clavicular fragmentation. To the best of our knowledge, this is the first case report addressing pellet embolization to the brachial artery.

\section{Case Report}

A 72 year old gentle man was admitted to the emergency department after he attempted suicide using a gun. The primary assessment followed the trauma protocol and revealed a gunshot injury to the right upper chest region with no pellet exit wound. There was no neurological deficits, active bleeding, palpable thrill or audible bruit (indicative of arteriovenous fistula), or pulsatile mass. Radial pulses were palpable and symmetrical bilaterally. Since the patient was hemodynamically stable, further radiological investigations were performed. A chest radiograph and computer tomography scan of the neck and chest were performed. These revealed the right clavicle comminuted fracture which, on consultation from the orthopedic team, was advised to manage conservatively and five pellets, lodged in the soft tissues of the right upper chest region. Because of the wound proximity to the major vessels, conventional angiography was carried out to exclude occult vascular injury. Angiography demonstrated a traumatic subclavian artery lesion and a foreign body in the brachial artery (Figure 1).

The patient was taken to the operating theatre for a surgical exploration. Before surgery the patient was re-examined and the right radial pulse was absent and the arm was cold, with reduced capillary refill. Viability of the extremity after trauma was assessed with The Mangled Extremity Severity Score (MESS), which estimated score was 6 ( 6 or less means that a limb is still salvageable). Under general anesthesia, a transverse infraclavicular incision approach was used, which revealed associated damage to the right subclavian vein by the clavicle fracture fragments. Venous injuries were treated with primary closure. Clavicle fractures and five pellets were removed. The right subclavian artery had suffered anterior and posterior penetrating injuries. Following systemic heparinization (5000 IU), the defect was repaired using an interposition saphenous vein graft. Exposure of the brachial artery was performed through a longitudinal incision in the cubital fossa. An embedded pellet (Figure 2) from the brachial artery was eliminated through a separate arteriotomy. Right arm distal pulses were restored. 


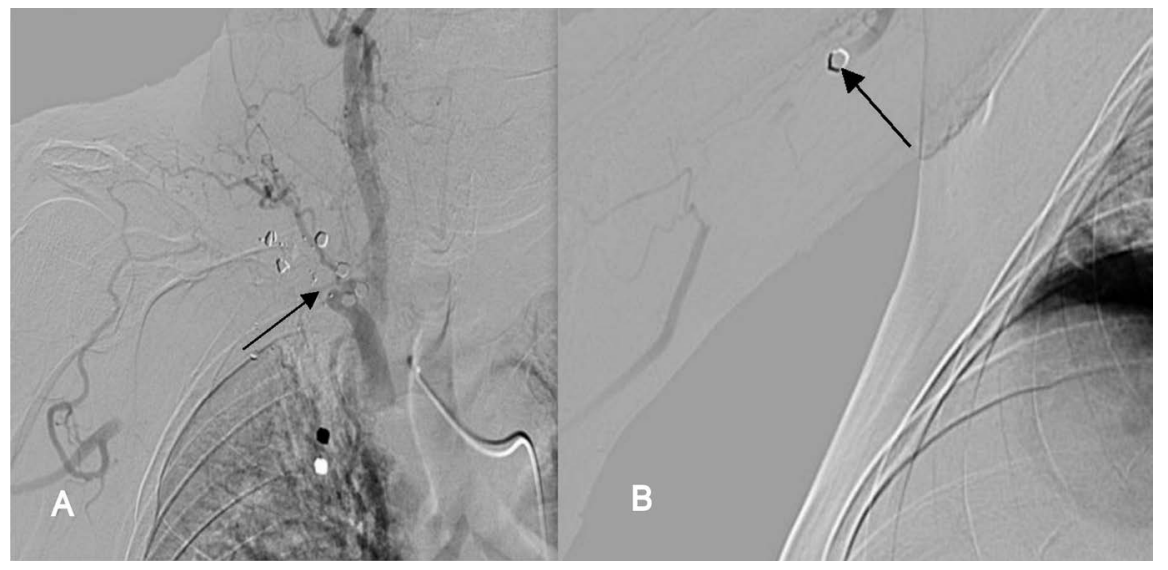

Figure 1. (A) A right subclavian artery traumatic injury caused by direct trauma mechanism; (B) Angiography shows right brachial artery distal embolization by pellet.

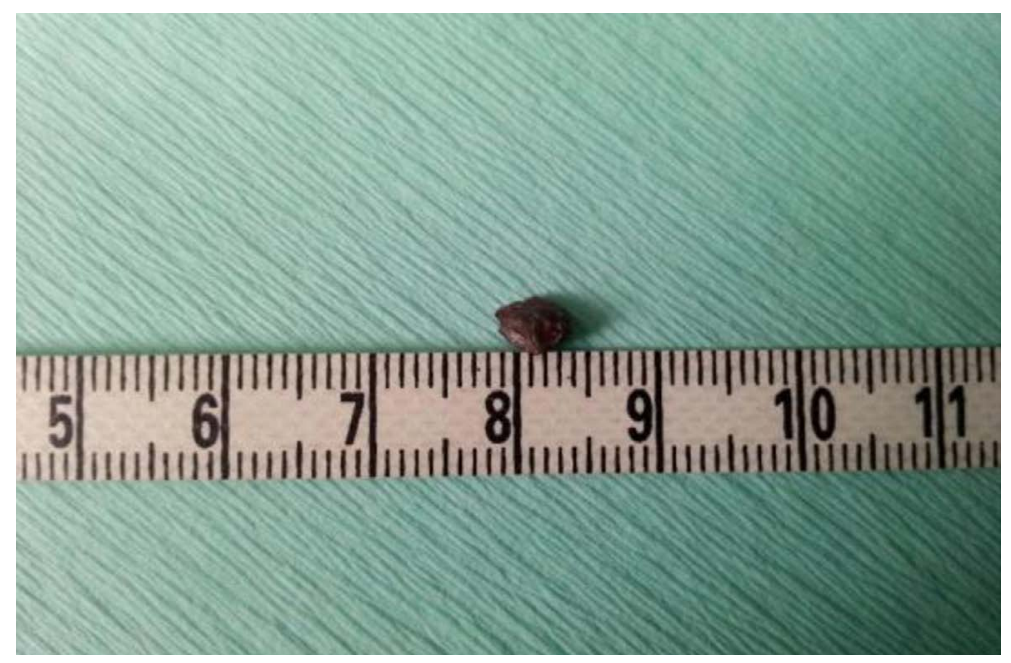

Figure 2. Pellet after removal from the brachial artery.

Control angiography was performed and no missed arterial injuries were found (Figure 3). Heparin was continued for 3 days (5000 IU three times a day) post operation. Intravenous antibiotic prophylaxis with cefuroxime and metronidazole was administered for 3 days as well. The patient was discharged on postoperative day 7 with normal motor function and no neurological deficits and remained well at one year follow-up with no reported complications.

\section{Discussion}

The majority of the vascular injuries are caused by penetrating trauma (90.2\%) with the commonest due to a low-velocity gunshot injury (56.4\%). Among all vascular injuries, incidence of upper extremity vascular injury is $50 \%$, and $25 \%$ of these involve the axillary and subclavian vessels [5]. Embolization by foreign body after a gunshot in the civilian setting is rare and the exact incidence is unknown. It is commoner in arteries (80\%) than veins (20\%) [6]. Drapanas T. et al. [4] study showed that the majority of the patients with arterial injury have other 


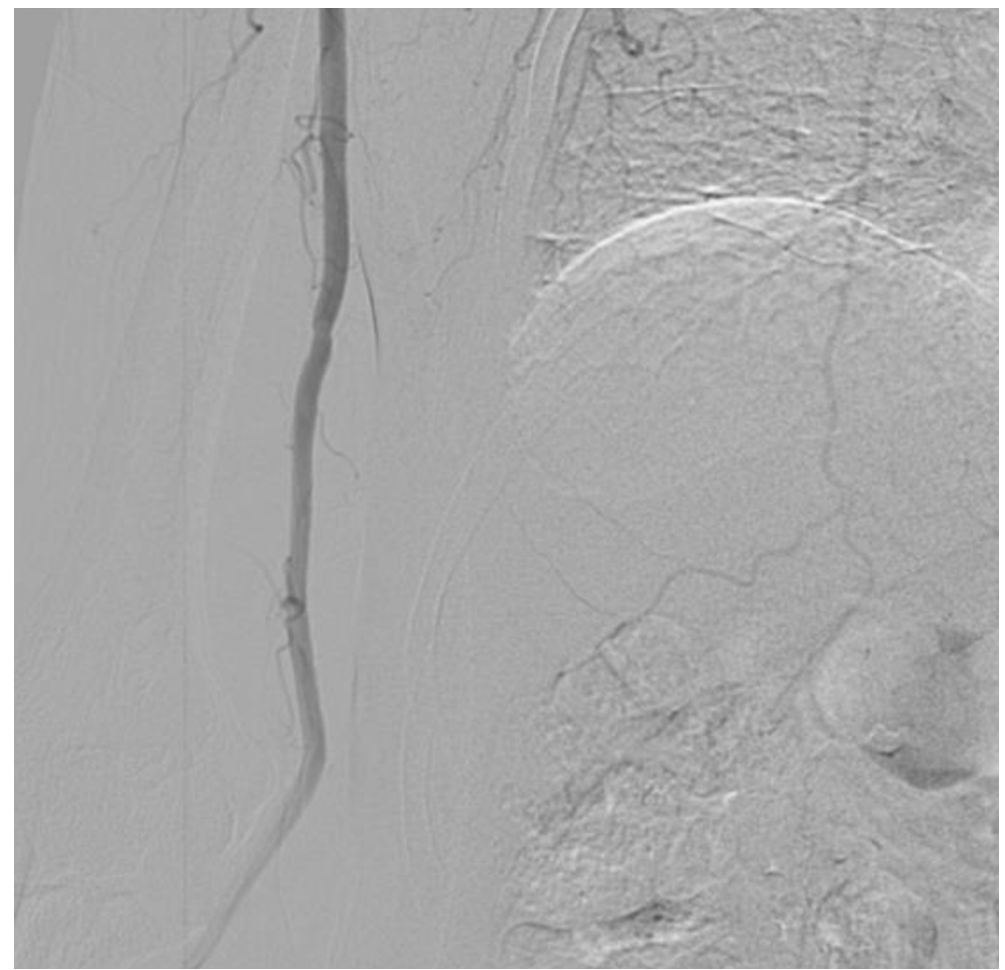

Figure 3. Postoperative angiography indicates normal blood flow to the upper limb arteries.

associated injuries. Among the 226 patients, $41.1 \%$ were found to have injuries to adjacent veins, $10 \%$ had neurological deficits with decreased motor function and $8.3 \%$ of the patients with extremity injuries had long bone fractures. In the study of penetrating subclavian artery injuries by Lin P.H. et al. [1], the commonest associated injury was that of the subclavian vein $44 \%$, followed by the brachial plexus $31 \%$, latter accounts for the majority of long term morbidity. Similar to our case, additionally to a right subclavian artery injury, the patient had clavicular comminuted fracture and bone fragments subsequently caused a subclavian vein injury.

Gunshots, classified as low velocity, are more frequently used in civilian settings as it was used in our case. When a low velocity bullet or pellet passes throw the tissues, it loses its kinetic energy and can remain lodged or enter the vessel. Within the vessel, the pellet usually migrates distally with blood flow to a site remote to the entry site, thus becoming an embolus and embeds in the site of the same vessel diameter as the pellet caliber is which results in distal ischemia [7].

The diagnosis of penetrating extremity vascular injuries is challenging. According to Drapanas T. et al. [5] study of 128 patients with injuries to major arteries of extremities, $27.3 \%$ had palpable pulses distal to the injury, which indicates that physical examination is not a good predictor of vascular injury. Although, more proximal vascular injuries may present with palpable distal pulses up to $70 \%$, secondary to a good collateralization at the shoulder or axilla [8]. It is therefore recommended to discharge patient with only "soft signs" of vascular 
injury, symmetrical pulses and normal $(<0.9)$ ABPI with a need of further surveillance [4]. In the present case, the patient exhibited with symmetrical radial pulses and had only "soft signs" of vascular injury, like wound proximity to the major vessels. Following admission to the operating theater he developed "hard signs" of acute artery occlusion-right radial pulse was lost and his arm was cold and pale. Without routine angiography, diagnosis could be delayed, hence culminating in unfavorable outcomes. Even when there is no signs of vascular injury and especially when there is no exit wound, embolization should be suspected and careful investigation should be performed.

For hemodynamically stable patients with penetrating gunshot injuries, conventional angiography, CT angiography or Duplex ultrasound are all recommended imaging modalities to perform routinely in order to evaluate possible vascular injury [7]. Conventional angiography is considered as a "gold standard" in diagnosis of penetrating vascular injuries. It can be also used for subsequent endovascular treatment. Angiography provides an accurate depiction of vascular lesions and assists for further surgery panning. It also helps distinguishing between intimal disruption and vasospasm by using vasodilators. Moreover, angiography can evaluate arterial dissection most accurately. However, because of its invasive nature, complications like hematoma, infection, vessel injury and etc. may occur [9]. CTA in comparison with DSA has a high sensitivity (96.2\%) and specificity (99.2\%) to diagnose vascular injuries in the extremities. CTA is less time consuming than DSA, which is very important factor in emergencytrauma setting [10]. According to Bynoe R.P. et al. [11] Duplex ultrasound has 95\% sensitivity and 99\% specificity to diagnose vascular injuries. There are some disadvantages related with this imaging modality, including the need of experienced examiner, 24 hour availability and compromised visualization in some areas like axilla and arterial bifurcation [3].

In the management of arterial injuries, which are considered as contaminated wounds, autologous saphenous vein from the non injured extremity is recommended as first choice conduit to replace the injured segment [12]. When autologous grafts can't be used, synthetic vascular grafts are an alternative. However, up to date there is no definitive data regarding the choice of vascular graft in the trauma setting. Feliciano D.V. et al. study included 206 patients with vascular wounds which revealed that prosthetic grafts are associated with lower patency rates compared with autologous vein grafts, however synthetic grafts didn't have any infectious complications, except the cases when the graft was exposed or was next to the site of osteomyelitis [13]. Controversies among the studies remains as some of them are showing identical results with autologous vein and synthetic grafts [14].

Extraction of intra-arterial bullets in the arterial system is mandatory if the patient exhibits symptoms of acute peripheral ischemia. Asymptomatic arterial and venous embolus by foreign body removal should be considered individually, according to the risk of possible displacement and further embolization [15]. We 
used local arteriotomy instead of catheter embolectomy to avoid the risk of iatrogenic intimal lesions.

Johnson CA [16] described successful results of endovascular repair of an injured subclavian artery in cases of blunt trauma and penetrating injuries. The ideal patients for this treatment option are those with low velocity trauma. However, this is not possible in all cases of axillo-subclavian injuries. If debridement of the wound is necessary, open surgical exposure is mandatory.

Complications after penetrating extremities injuries are devastating. In Drapanas T. et al. [5] study, morbidity after surgery, was even higher than the mortality. From the 181 patients with injuries to arteries of the extremities the amputation rate was $7.1 \%, 20.4 \%$ had decreased limb function due to motor nerve deficits or muscle damage, $10.2 \%$ complained of chronic pain, infection occurred for $5 \%$ of the patients and $23 \%$ had operated extremity edema. Luckily, our patient suffered no postoperative complications and no sequelae.

\section{Conclusion}

We report an extremely rare case of pellet embolization to the brachial artery following a thoracic low-velocity gunshot injury. This case highlights the need for further vascular imaging even when clinical examination is normal, to rule out the presence of subtle vascular injuries.

\section{Grant Support and Disclosure of Financial Arrangements}

All authors declare no grants or financial support.

\section{Acknowledgements}

Appreciation is expressed to Dr. Agnè Gimžauskaite for her advice in the manuscript preparation.

\section{References}

[1] Lin, P.H., Koffron, A.J., Guske, P.J., et al. (2003) Penetrating Injuries of the Subclavian Artery. American Journal of Surgery, 185, 580-584. https://doi.org/10.1016/S0002-9610(03)00070-9

[2] Demetriades, D., Rabinowitz, B., Pezikis, A., et al. (1987) Subclavian Vascular Injuries. British Journal of Surgery, 74, 1001-1003. https://doi.org/10.1002/bjs.1800741114

[3] Burg, A., Nachum, G, Salai, M., et al. (2009) Treating Civilian Gunshot Wounds to the Extremities in a Level 1 Trauma Center: Our Experience and Recommendations. Israel Medical Association Journal, 11, 546-551.

[4] Feliciano, D.V., Moore, F.A., Moore, E.E., et al. (2011) Evaluation and Management of Peripheral Vascular Injury. Part 1. Western Trauma Association/Critical Decisions in Trauma. The Journal of Trauma, 70, 1551-1556. https://doi.org/10.1097/TA.0b013e31821b5bdd

[5] Drapanas, T., Hewitt, R.L., Weichert 3rd, R.F., et al. (1970) Civilian Vascular Injuries: A Critical Appraisal of Three Decades of Management. Annals of Surgery, 172, 351-360. https://doi.org/10.1097/00000658-197009000-00005 
[6] Colquhoun, I.W., Jamieson, M.P. and Pollock, J.C. (1991) Venous Bullet Embolism: A Complication of Airgun Pellet Injuries. Scottish Medical Journal, 36, 16-17. https://doi.org/10.1177/003693309103600107

[7] Martin, M.J. and Long, W.B. (2014) Vascular Trauma: Epidemiology and Natural History. In: Cronenwett, J.L. and Johnston, K.W., Eds., Rutherford's Vascular Surgery, Vol 2, 8th Edition, Saunders, Philadelphia, 2422-2437.

[8] Zelenock, G.B., Kazmers, A., Graham, L.M., et al. (1985) Nonpenetrating Subclavian Artery Injuries. Archives of Surgery, 120, 685-692. https://doi.org/10.1177/003693309103600107

[9] Ravari, H., Pezeshki Rad, M., Bahadori, A., et al. (2014) Comparison of Conventional Angiographic Findings between Trauma Patients with or without Runoff. Bulletin of Emergency \& Trauma, 2, 72-76.

[10] Jens, S., Kerstens, M.K., Legemate, D.A., et al. (2013) Diagnostic Performance of Computed Tomography Angiography in Peripheral Arterial Injury Due to Trauma: A Systematic Review and Meta-Analysis. European Journal of Vascular and Endovascular Surgery, 46, 329-337. https://doi.org/10.1177/003693309103600107

[11] Bynoe, R.P., Miles, W.S., Bell, R.M., et al. (1991) Noninvasive Diagnosis of Vascular Trauma by Duplex Ultrasonography. Journal of Vascular Surgery, 14, 346-352.

[12] Keen, R.R., Meyer, J.P., Durham, J.R., et al. (1991) Autogenous Vein Graft Repair of Injured Extremity Arteries: Early and Late Results with 134 Consecutive Patients. Journal of Vascular Surgery, 13, 664-668.

[13] Feliciano, D.V., Mattox, K.L., Graham, J.M., et al. (1985) Five-Year Experience with PTFE Grafts in Vascular Wounds. The Journal of Trauma, 25, 71-82. https://doi.org/10.1097/00005373-198501000-00012

[14] Degiannis, E., Levy, R.D., Potokar, T., et al. (1995) Penetrating Injuries of the Axillary Artery. ANZ Journal of Surgery, 65, 327-330. https://doi.org/10.1111/j.1445-2197.1995.tb00647.x

[15] Michelassi, F., Pietrabissa, A., Ferrari, M., et al. (1990) Bullet Emboli to the Systemic and Venous Circulation. Surgery, 107, 239-245.

[16] Johnson, C.A. (2010) Endovascular Management of Peripheral Vascular Trauma. Seminars in Interventional Radiology, 27, 38-43.

https://doi.org/10.1055/s-0030-1247887 